\title{
A semi-supervised deep learning approach for predicting the functional effects of genomic non-coding variations
}

\author{
Hao Jia', Sung-Joon Park ${ }^{1,2}$ and Kenta Nakai ${ }^{1,2^{*}}$ (i)
}

From 19th International Conference on Bioinformatics 2020 (InCoB2020)

Virtual. 25-29 November 2020

\author{
*Correspondence: \\ knakai@ims.u-tokyo.ac.jp \\ ${ }^{1}$ Department of Computer \\ Science, The University \\ of Tokyo, 7-3-1 Hongo, \\ Bunkyo-ku, Tokyo 113-8656, \\ Japan \\ Full list of author information \\ is available at the end of the \\ article
}

\begin{abstract}
Background: Understanding the functional effects of non-coding variants is important as they are often associated with gene-expression alteration and disease development. Over the past few years, many computational tools have been developed to predict their functional impact. However, the intrinsic difficulty in dealing with the scarcity of data leads to the necessity to further improve the algorithms. In this work, we propose a novel method, employing a semi-supervised deep-learning model with pseudo labels, which takes advantage of learning from both experimentally annotated and unannotated data.

Results: We prepared known functional non-coding variants with histone marks, DNA accessibility, and sequence context in GM12878, HepG2, and K562 cell lines. Applying our method to the dataset demonstrated its outstanding performance, compared with that of existing tools. Our results also indicated that the semi-supervised model with pseudo labels achieves higher predictive performance than the supervised model without pseudo labels. Interestingly, a model trained with the data in a certain cell line is unlikely to succeed in other cell lines, which implies the cell-type-specific nature of the non-coding variants. Remarkably, we found that DNA accessibility significantly contributes to the functional consequence of variants, which suggests the importance of open chromatin conformation prior to establishing the interaction of non-coding variants with gene regulation.
\end{abstract}

Conclusions: The semi-supervised deep learning model coupled with pseudo labeling has advantages in studying with limited datasets, which is not unusual in biology. Our study provides an effective approach in finding non-coding mutations potentially associated with various biological phenomena, including human diseases.

Keywords: Non-coding variants, Epigenome, Semi-supervised learning, Deep learning, Pseudo label author(s) and the source, provide a link to the Creative Commons licence, and indicate if changes were made. The images or other third party material in this article are included in the article's Creative Commons licence, unless indicated otherwise in a credit line to the material. If material is not included in the article's Creative Commons licence and your intended use is not permitted by statutory regulation or exceeds the permitted use, you will need to obtain permission directly from the copyright holder. To view a copy of this licence, visit http:// creativecommons.org/licenses/by/4.0/. The Creative Commons Public Domain Dedication waiver (http://creativecommons.org/publi cdomain/zero/1.0/) applies to the data made available in this article, unless otherwise stated in a credit line to the data. 


\section{Background}

It is well known that more than $95 \%$ of the human genome is non-coding DNA sequences that do not encode proteins [1]. Recently, many studies have found that these non-coding sequences play an indispensable role in biology. For example, genome-wide association studies have identified that the majority of variant loci $(88 \%)$ associated with human diseases lie in non-coding regions and modulate gene regulation in a tissue- or cell-type-specific manner [2]. Some non-coding mutations introduce the gain and loss of functions of transcription factor binding sites [3], and the epigenomic modifications studied by large projects, such as Encyclopedia of DNA Elements (ENCODE) [4] and Roadmap Epigenomics [5], co-exist with the non-coding variants that are associated with diseases and traits.

For understanding the functional consequence of non-coding genetic variations, many researchers have utilized distinctive explanatory features and proposed computational tools. For example, FUN_LDA [6] is an unsupervised latent Dirichlet allocation model, and GenoSkyline [7] is trained by a two-component probabilistic mixture model. These two approaches calculate the prediction scores using histone modifications and DNase I hypersensitivity. Eigen [8] applies one unsupervised spectral learning method, and deltaSVMs [9] is a support vector machine (SVM) derived by the gkm-SVM classifier for the effective prediction of regulatory variants. CADD [10], a linear kernel SVM algorithm, and DANN [11], a deep learning model, utilize the same feature sets each other. DeepSEA [12], a deep learning-based framework, learns from sequence patterns in non-coding regions to predict allele-specific chromatin profile.

Over the past few years, the unsupervised machine learning and deep learning (DL) methods above mentioned have been successfully applied to this issue. However, these approaches rely on the input dataset and is refractory to the growth of its data scale [13]. In this context, many laborious experiments performed have ignored the fact that the number of non-coding variants experimentally validated is much fewer compared with millions of variants across the genome.

In this study, we propose a novel method employing a semi-supervised DL model with pseudo labels. To overcome the scarcity of available data, our method takes advantage of learning from both labeled and unlabeled data. Furthermore, we utilize epigenetic annotations and sequence features, which are observed from the genomic regions of non-coding variants to infer the important factors for the functional consequence.

\section{Results}

\section{Overall structure of the proposed DL model}

Recently, semi-supervised learning (SSL) has been extensively studied and has become more popular in various research fields [14-16]. In particular, the SSL coupled with pseudo labels, providing high-quality pseudo labels for unlabeled largescale data during training, has been proven to allow the neural networks to make more confident predictions [17-19]. Taking its advantage, we developed an SSL model for analyzing genetic and epigenetic signatures in the 150-bp genomic regions where non-coding mutations occurred, as shown in Fig. 1. 


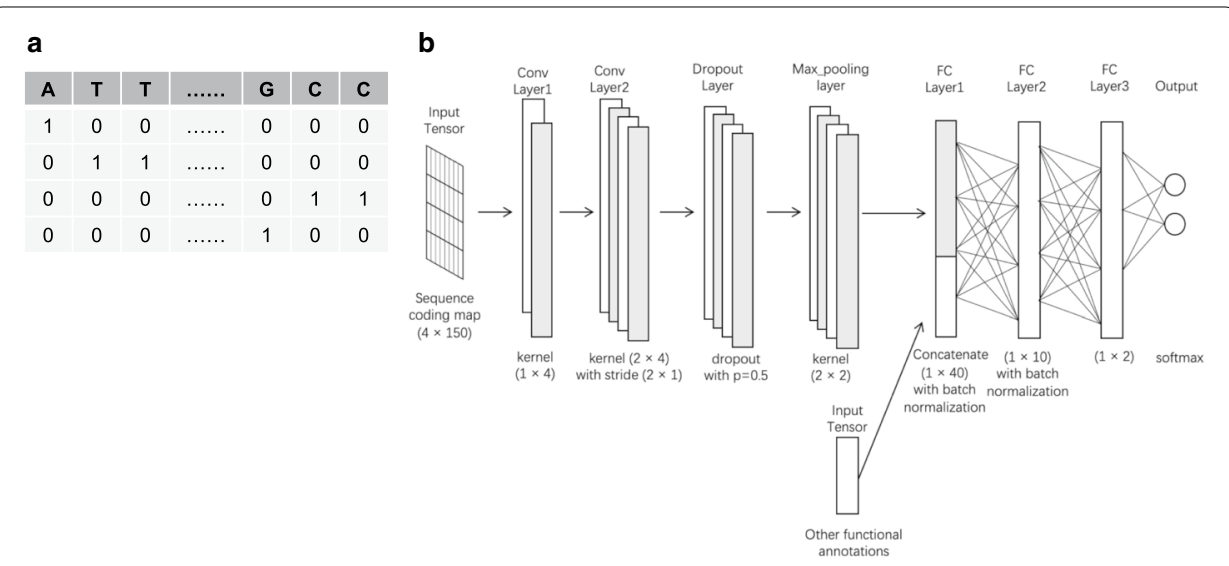

Fig. 1 An overview of the deep neural network used in this study. a Sequence coding map of a 150-bp region centered by a non-coding variant locus. $\mathbf{b}$ Schematic representation of our network architecture. FC, fully connected

As an input to our DL neural network, the nucleotides of a 150-bp region, centered by any non-coding variant locus, are represented by the binary vectors, as known as One-hot Encoding (Fig. 1a). Simultaneously, the 150-bp regions are scored by three scoring functions (i.e. Peak, Max, and Sum), measuring 10 histone enrichments and DNase sensitivity. The 10 different types of nucleotide compositions are also measured. These epigenetic and nucleotide composition features are concatenated with the output of the max-pooling function in our neural network structure (Fig. 1b).

\section{Predictive performance of our model and inference of impactful features}

To test the feasibility of our approach, we downloaded the non-coding variant loci known in the human cell lines (GM12878, HepG2, and K562) [20]. Since these cell lines have been assayed extensively in ENCODE, we could access large-scale genomic and epigenomic data, which can be used to characterize the loci on a genomic scale.

First, we investigated the landscape of the input feature maps. In K562 cell line, the Max and Sum scores for the epigenetic marks showed broad ranges of distribution, and their patterns were greatly similar each other (Fig. 2a). These scores were similarly correlated with the distribution of non-coding variants, which was not observed in nucleotide composition features (Fig. 2b). Interestingly, the feature of DNase sensitivity was strongly correlated with the non-coding variants in all cases. Next, we assessed the performance of our model using the datasets shown in Table 1. As a result, although its performance in AUC reached to 0.75 in GM12878, no drastic differences were observed among cell lines (Fig. 2c).

To examine what features contributed more to the performance, we grouped the epigenetic features into 6 functional categories; (I) Enhancer: H3K4me1 and H3K27ac, (II) Promoter: H3K4me2, H3K4me3 and H3K9ac, (III) Structural marks: H3K36me3 and H3K79me2, (IV) Heterochromatin: H3K9me3, H3K27me3, (V) Transcribed gene-body: H4K20me1, and (VI) DNA accessibility: DNase I sensitivity. As shown in Fig. 2d, the Max-score-based models with each of group I, group II, and group VI showed higher AUC values, consistent with the distribution of 


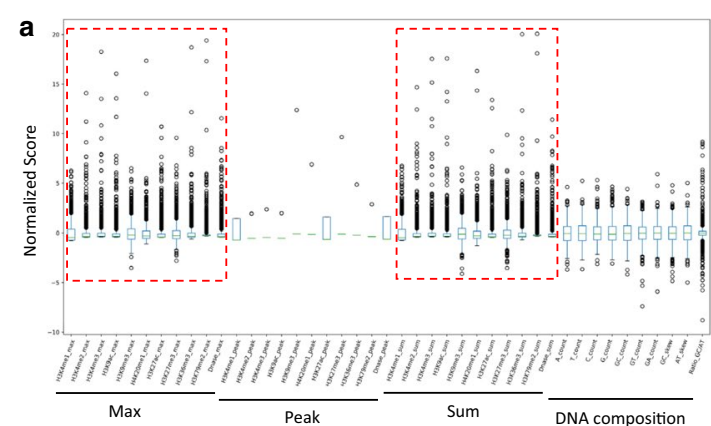

b

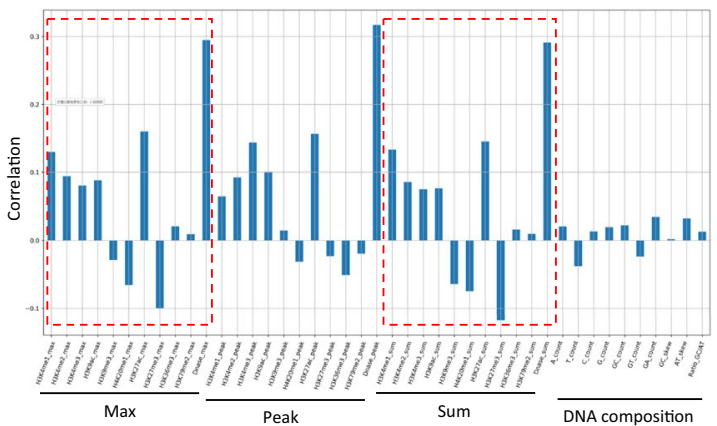

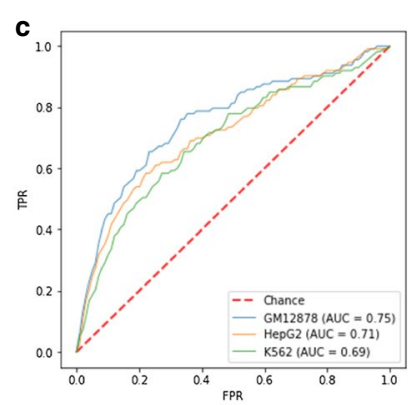

d

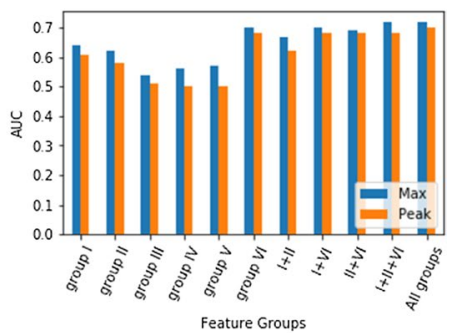

Fig. 2 Feature distribution and the predictive performance of our deep learning model. a Plots showing the distribution of each score in the input feature map after preprocessing for K562. b Pearson correlation of each feature vector with the labels of non-coding variants in K562. c ROC curves showing the performance of our model. d AUC values showing the performance of our model with each of the grouped features in GM12878. $T P R$ true positive rate, FDR false discovery rate, AUC area under the ROC (receiver operating characteristic) curve, group / histone marks on enhancers, group // histone marks on promoters, group III structural histone marks, group IV heterochromatin histone marks, group $V$ histone marks on transcribed gene-body, group VI DNA accessibility assayed by DNase I sensitivity

Table 1 Experimentally labeled non-coding variant loci in three human cell lines

\begin{tabular}{llll}
\hline Cell lines & Positive & Negative & Total \\
\hline Lymphoblastoid (GM12878) & 683 & 2745 & 3428 \\
Liver carcinoma (HepG2) & 523 & 1432 & 1955 \\
Erythroleukemia (K562) & 340 & 1356 & 1696
\end{tabular}

Positive a locus affecting gene expression, negative a locus showing no effect

Pearson correlation in Fig. 2b. Remarkably, DNA accessibility (i.e. group VI) largely contributed to the performance. In contrast, nucleotide-based features, such as the GC-count, were less effective (Additional file 1: Fig. S1), in which the weak Pearson correlation in Fig. 2b may also explain this result.

Taken together, the epigenetic annotations, particularly DNA accessibility, are more explanatory for the presence of functional non-coding variants in K562. This result was also observed in GM12878 and HepG2 (Additional file 1: Fig. S2 and Fig. S3). 


\section{Comparing with other models}

We compared our semi-supervised learning by a deep neural network with pseudo labels (SSL_dnn) with seven existing unsupervised models; FUN-LDA [6], GenoSkyline [7], Eigen [8], deltaSVM [9], CADD [10], DANN [11], and DeepSEA [12]. Due to the technical difficulties of implementing these classifiers, we downloaded their prediction scores for each non-coding variant locus from the previous study [20] that performed the prediction with the classifiers and the three cell lines. By applying SSL_dnn to the same validation dataset, we could draw AUC curves and compare them. As a result, SSL_dnn exhibited higher AUC values; 0.75 in GM12878, 0.71 in HepG2, 0.69 in K562 (Fig. 3a-c).

Next, we compared SSL_dnn with a supervised deep neural network without pseudo labels. Their architectures and running parameters were exactly the same, but the supervised model considered only the supervised loss during the training process. As shown in Fig. 3d, although the classifiers showed similar growth trends for AUC values at the beginning, SSL_dnn gradually obtained better performance as the epoch increased in

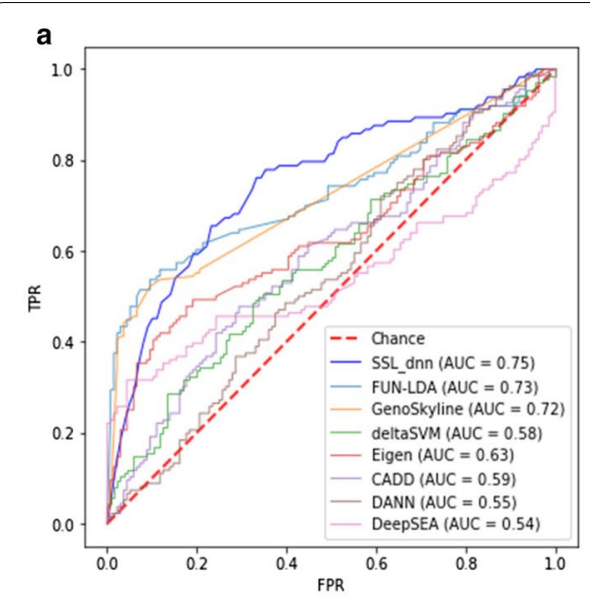

\section{b}
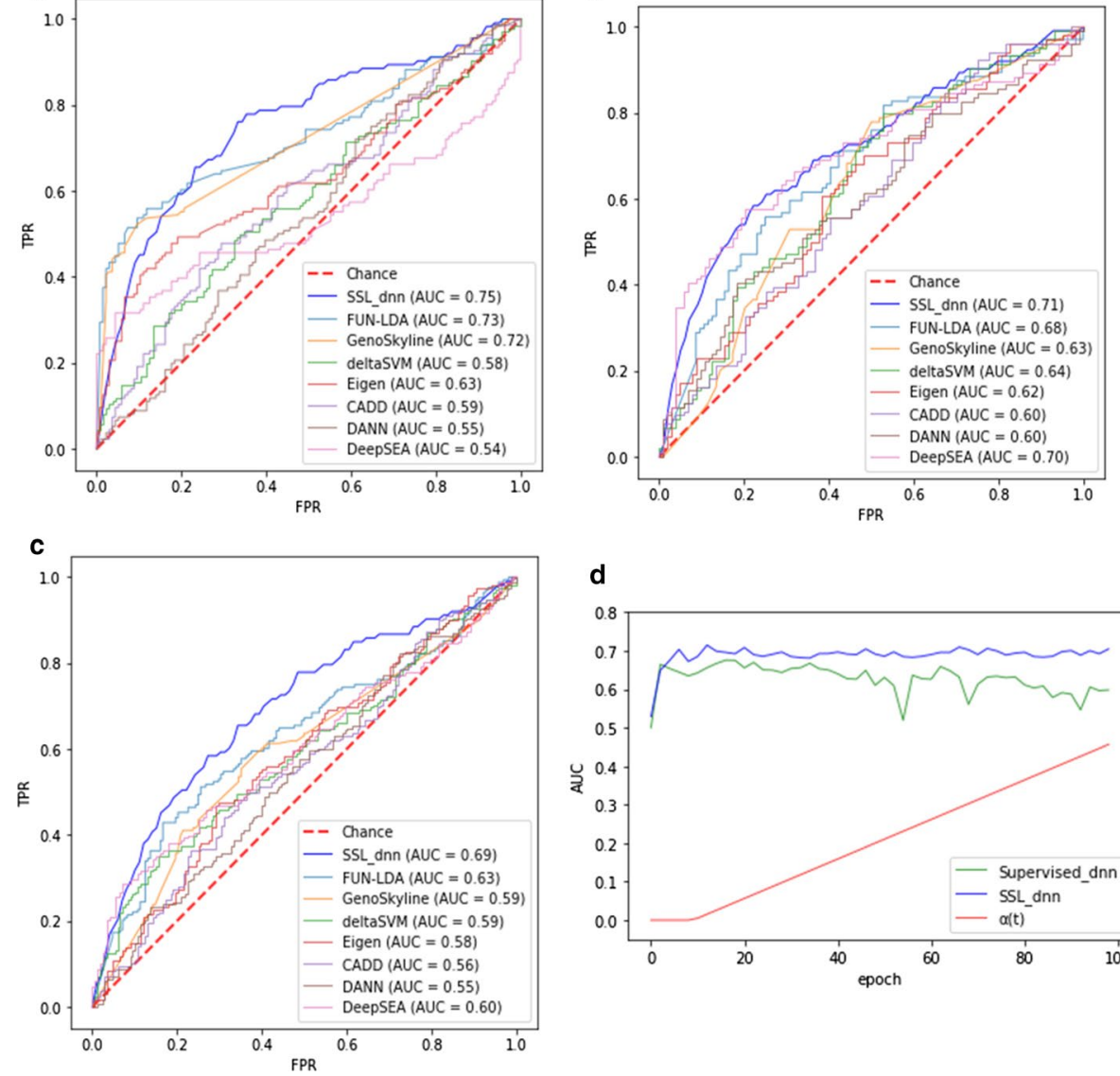

\section{d}

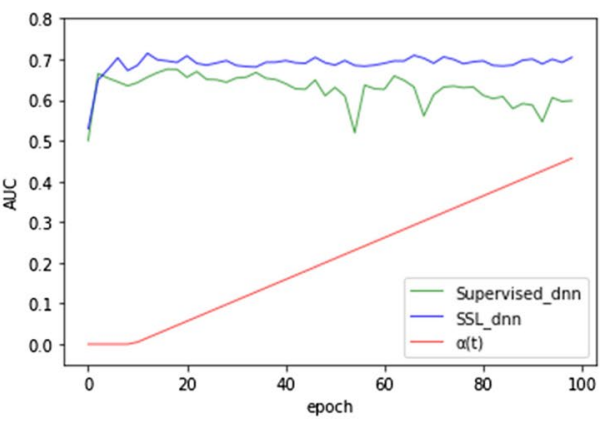

Fig. 3 Comparing the performance of the proposed deep learning model with existing models and a supervised model. a ROC curve in GM12878 dataset. b ROC curve in HepG2 dataset. c ROC curve in K562 dataset. $\mathbf{d}$ AUC values showing the performance of the proposed model and a supervised model without pseudo labels in K562 dataset. AUC, area under the ROC (receiver operating characteristic) curve; Supervised_ dnn, deep neural network; SSL_dnn, semi-supervised learning by dnn with pseudo labels; $\alpha(t)$, a parameter in the loss function 
K562 cell line. It is expected that the two classifiers are practically the same algorithms at the beginning since the cross-entropy loss function in SSL_dnn composes only labeled loss [i.e. $\alpha(t)=0$ ]. When the dynamic schedule of $\alpha(t)$ starts incorporating unlabeled loss in the cross-entropy loss function, the performance of SSL_dnn is outstanding, which suggests the impactful contribution of pseudo labels.

Consequently, we confirmed that the proposed model outperforms the current unsupervised models and supervised ones without pseudo labels in terms of AUC value, utilizing both experimentally confirmed labeled data and a large amount of unlabeled data.

\section{Predicting non-coding variants in specific cell lines}

To investigate whether the nature of non-coding variants is cell-type specific or promiscuous, we trained SSL_dnn with the dataset of a certain cell line and predicted the validation datasets of other cell lines. Then, we evaluated its performance using AUC as well as Accuracy, the fraction of correctly predicted labels in total loci. As shown in Fig. 4, the models did not exhibit satisfactory predictive performance for the variant loci in other cell lines that are not used for the training. This result suggests that the noncoding variants are involved in cell-type specification, accompanied by different variant loci and distinctive histone modifications.

\section{Discussion}

It is known that pseudo labeling helps to exploit the prediction of the DL model with ground truth for unlabeled data and also enables the DL to learn from the unlabeled data. This algorithmic uniqueness has given a new window to study various biological phenomena with smaller numbers of experimentally confirmed data and a large amount of relevant data unannotated. In this study, we developed a semi-supervised DL model with pseudo labels to predict the functional effects of non-coding variants.

We confirmed that the deep neural network exploits the pseudo labels assigned for unlabeled data and the labeled data updated with these pseudo labels during the training process. After setting up fair comparisons as possible, our approach was prominent
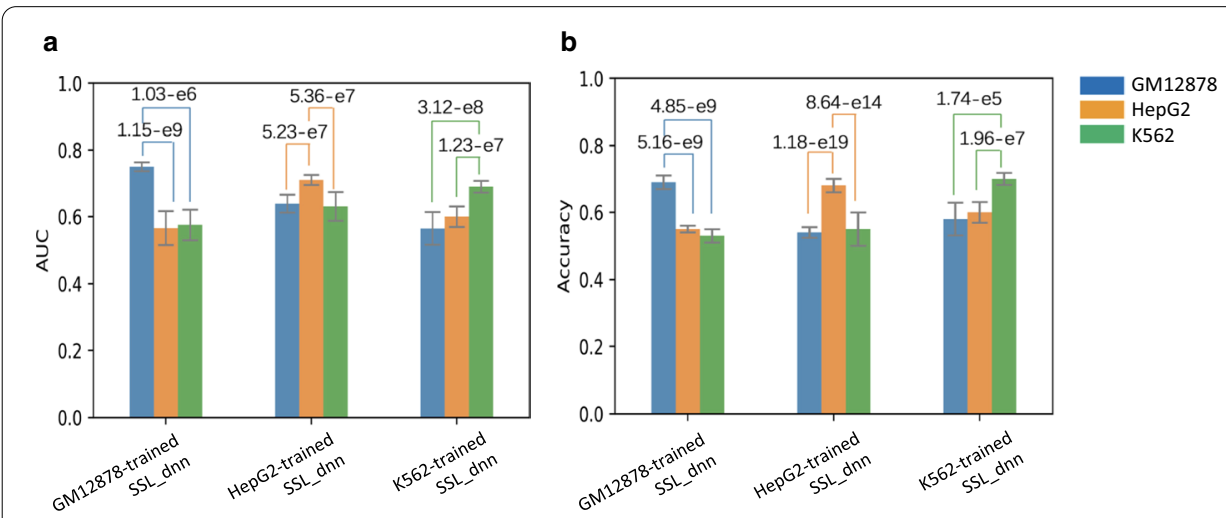

Fig. 4 The performance of the proposed model trained with a certain cell-line dataset and evaluated with the validation datasets of other cell lines by AUC values (a) and Accuracy values (b). The bars represent the standard errors in fivefold cross validation and $p$-values were calculated by two-tailed $t$-test; AUC, area under the ROC (receiver operating characteristic) curve; SSL_dnn, semi-supervised learning by a deep neural network with pseudo labels 
Table 2 Training and validation datasets prepared from the loci in Table 1

\begin{tabular}{llll}
\hline Cell lines & \multicolumn{2}{l}{ Training } & \multirow{2}{*}{ Validation (pos/neg) } \\
\cline { 2 - 3 } & Labeled (pos/neg) & Unlabeled (pos/neg) & \\
\hline Lymphoblastoid (GM12878) & $550(275 / 275)$ & $2606(272 / 2,334)$ & $272(136 / 136)$ \\
Liver carcinoma (HepG2) & $450(225 / 225)$ & $1297(197 / 1,103)$ & $208(104 / 104)$ \\
Erythroleukemia (K562) & $350(175 / 175)$ & $1210(97 / 1,113)$ & $136(168 / 168)$ \\
\hline
\end{tabular}

pos a positive locus affecting gene expression, neg a negative locus showing no effect

compared to the existing unsupervised classifiers and the supervised classifier without using pseudo labels under the same setting. Of note, we used the imbalanced number of positive and negative data in the unlabeled datasets (Table 2). When adding the imbalanced unlabeled data to the training process, the performance of our model became higher than that of the supervised classifier (Fig. 3d). This result indicates that the imbalance of the training dataset may not negatively affect the performance of SSL, which requires further detailed studies.

Through the investigation of impactful features for the prediction, we found that DNA accessibility reflecting open chromatin status [21] is the most indispensable feature (Fig. 2). This feature exhibited a relatively higher correlation with the distribution of functional non-coding variants. In contrast, the features based on nucleotide compositions were less effective. Importantly, our model trained with the dataset in a certain cell line is unlikely to succeed in predicting the variants in other cell lines. These results suggest that cell-type-specific epigenetic factors related to open chromatin conformation interplay with the functional non-coding variants.

We here employed the experimental annotations and epigenomic data in human cell lines, which allowed conducting the validation of our method and the characterization of the non-coding variations on a genomic scale. As future works, the extensive assessment with human disease samples and the incorporation of more comprehensive annotations require, which will give insights into how and why the non-coding variants are involved in diseases and traits.

\section{Conclusions}

The semi-supervised deep learning model coupled with pseudo labeling has advantages in studying with limited datasets, which is not unusual in biology. Our study provided an effective approach to find non-coding mutations potentially associated with various biological phenomena including human diseases.

\section{Methods}

Preparing datasets

We downloaded the non-coding variant loci and their labels in GM12878, HepG2, and K562 cell lines from a previous study [20]: label 1 for positive loci affecting the gene regulation and label 0 for negative loci that are nothing to do with gene expression (Table 1). In addition, we downloaded the processed datasets of histone modifications and DNase I sensitivity from ENCODE. The histone ChIP-seq data included H3K4me1, H3K4me2, 
H3K4me3, H3K9ac, H3K9me3, H4K20me1, H3K27ac, H3K27me3, H3K36me3, and H3K79me2.

\section{Generating feature vectors}

After checking the overlap of non-coding loci with the epigenetic marks, we generated three types of feature vectors: (1) Peak, 1 for a non-coding variant locus positioned within a peak region of an epigenetic mark, zero for others; (2) Max, the maximum enrichment score within a 150-bp region centered by a non-coding variant locus; (3) Sum, the sum of enrichment score for the 150-bp region. We calculated the nucleotide compositions of the 150-bp region: (4) Mononucleotide count, A_count (Adenine), T_count (Thymine), G_count (Guanine), and C_count (Cytosine); (5) Dinucleotide count, GC_count, GT_ count, and GA_count; (6) Skew, AT_skew [= (A_count - T_count)/AT_count] and GC_ skew [= (G_count - C_count)/GC_count]; (7) Ratio of skew (=GC_skew/AT_skew).In addition, we encoded each base in the 150-bp region by adopting the one-hot encoding; $[1,0,0,0]$ for $\mathrm{A},[0,1,0,0]$ for $\mathrm{T},[0,0,1,0]$ for $\mathrm{C}$, and $[0,0,0,1]$ for $\mathrm{G}$.

\section{Implementing a deep learning (DL) model}

Our DL model consists of two convolutional neural layers that deal with the code matrix with the shape of 150 (sequence length) $\times 4$ (size of one hot coding approach). The output channel sizes in the convolutional layers are 2 and 4, respectively. The first convolutional neural layer uses a $(1 \times 4)$ convolutional filter with no padding for the information extraction from nucleotide vocabularies, while the second one applies a $(2 \times 4)$ filter and a $(2 \times 1)$ stride step. We employed the dropout function as the third layer. This function randomly assigns zero for some hidden units, making them be omitted during training, which contributes to minimize overfitting [22]. We used a max-pooling layer with the kernel size of $(2 \times 2)$, reserving the maximum values in windows and leaving a dense feature map with the size $(4 \times 1 \times 72)$ to the next layer. We also used the ReLU (Rectifie Linear Units) function [23] as the activation method for each neural unit.

Our model included three fully-connected (FC) layers, which are also known as dense layers, with the sizes of 40,10 , and 2, respectively. The input to the first FC layer is generated by concatenating the output of the max pooling function with the additional feature map of the epigenetic and nucleotide composition features. We added the dropout function and the batch normalization function to the first and second FC layers, making nonlinear transformations for the incoming data [24]. After the third FC layer, we applied the ReLU activation function. The final output layer consists of two neural units that correspond to the probability of two classifications.

\section{Implementing a semi-supervised DL model with pseudo labels}

The concept of model training with pseudo labels for real unlabeled and large-scale data has been proved. As briefly, the prediction $\widehat{y_{l}}$ of a deep neural network is given by

$$
\widehat{y_{l}}:=\operatorname{argmaxf} f_{\theta}\left(\mathrm{x}_{\mathrm{i}}\right)_{\mathrm{j}},
$$

where $f_{\theta}$ is the function directly mapping the input space $x_{i}$ to confidence scores. The output is a two-dimensional vector for each input feature map. The network is trained by minimizing the cross-entropy loss L given by 


$$
\mathrm{L}=\mathrm{L}_{\text {label }}+\alpha \mathrm{L}_{\text {unlabel }}
$$

where $\alpha$ is a coefficient set by considering the tradeoff for labeled versus unlabeled conditions. The $\alpha$ at the current batch $t$ is defined by the dynamic function [23]; 0 when $t<T_{1}, \frac{t-T_{1}}{T_{2}-T_{1}}$ when $T_{1}<t<T_{2}$, otherwise 1 .

For training our deep neural network, we first divided the labelled dataset of a cell line into three parts: labeled and unlabeled datasets for training, and a validation dataset for testing (Table 2). In order to make the labeled dataset and validation dataset balanced, the remaining positive loci is much fewer than the negative loci in the unlabeled dataset. Using the training datasets, we performed the iterative training process by initializing with random parameters: the process with the labeled dataset was monitored by a supervised loss term, then the unlabeled dataset was predicted by the trained model. The class that had the maximum predicted probability in the twodimensional output vector was selected as the "real" label to train the model. Then, the cross-entropy loss was calculated for optimizing the model. Of note, the number of unlabeled data decreases during the iteration as the unlabeled data with the most confident pseudo-labels is added to the labeled datasets to be used in the next epoch.

\section{Parameter setting}

We used the stochastic gradient descent function [25] to update the parameters with the learning rate 0.03 . We set the mini-batch sizes for the labeled and unlabeled training datasets and for the validation dataset to 16,32 , and 20 , respectively. The threshold to select the confidential pseudo-labels was 0.95 . We used $T_{1}=100, T_{2}=600$.

Abbreviations

AUC: Area under ROC curve; DL: Deep learning; Encode: Encyclopedia of DNA Elements; ReLU: Rectifie Linear Unit; ROC: Receiver operating characteristic; SSL_dnn:: Semi-supervised learning by deep neural network with pseudo labels; SSL: Semi-supervised learning; SVM: Support vector machine.

\section{Supplementary Information}

The online version contains supplementary material available at https://doi.org/10.1186/s12859-021-03999-8.

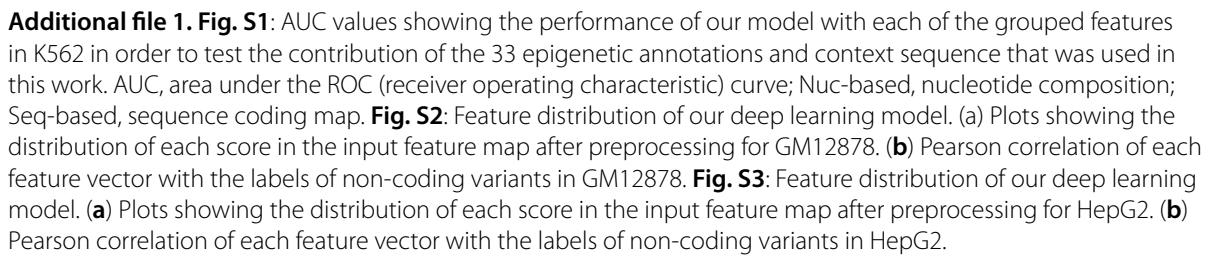
in $\mathrm{K} 562$ in order to test the contribution of the 33 epigenetic annotations and context sequence that was used in this work. AUC, area under the ROC (receiver operating characteristic) curve; Nuc-based, nucleotide composition; Seq-based, sequence coding map. Fig. S2: Feature distribution of our deep learning model. (a) Plots showing the distribution of each score in the input feature map after preprocessing for GM12878. (b) Pearson correlation of each feature vector with the labels of non-coding variants in GM12878. Fig. S3: Feature distribution of our deep learning model. (a) Plots showing the distribution of each score in the input feature map after preprocessing for HepG2. (b) Pearson correlation of each feature vector with the labels of non-coding variants in HepG2.

Acknowledgements

Computational resources were provided by the supercomputer system SHIROKANE at the Human Genome Center, the Institute of Medical Science, The University of Tokyo.

\section{About this supplement}

This article has been published as part of BMC Bioinformatics Volume 22 Supplement 6, 2021: 19th International Conference on Bioinformatics 2020 (InCoB2020). The full contents of the supplement are available online at https://bmcbioinformati cs.biomedcentral.com/articles/supplements/volume-22-supplement-6.

\section{Authors' contributions}

KN conceived of and designed the study. $\mathrm{HJ}$ and KN designed and performed all analyses. HJ, SJP and KN drafted the manuscript. All authors read and approved the final manuscript. 


\section{Funding}

This research was supported by Japan Society for the Promotion of Science (JSPS) KAKENHI (Grant No. 19H03213).

SJP was supported by JSPS KAKENHI (Grant No. 20K06606). The funding body had no direct roles in the design or execution of the study.

\section{Availability of data and materials}

The histone ChIP-seq and DNase I datasets were downloaded from the ENCODE repository at https://www.encod eproject.org/: E116 (GM12878), E1 18 (HepG2), and E123 (K562). The processed results are available either as supplementary data or upon request.

\section{Ethics approval and consent to participate}

Not applicable.

\section{Consent for publication}

Not applicable.

\section{Competing interests}

The authors declare that they have no competing interests.

\section{Author details}

${ }^{1}$ Department of Computer Science, The University of Tokyo, 7-3-1 Hongo, Bunkyo-ku, Tokyo 113-8656, Japan. ${ }^{2}$ Human Genome Center, the Institute of Medical Science, The University of Tokyo, 4-6-1 Shirokanedai, Minato-ku, Tokyo 108-8639, Japan.

Received: 21 January 2021 Accepted: 5 February 2021

Published: 2 June 2021

\section{References}

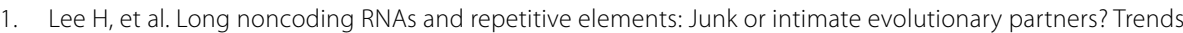
Genet. 2019. https://doi.org/10.1016/j.tig.2019.09.006.

2. Hindorff $L A$, et al. Potential etiologic and functional implications of genome-wide association loci for human diseases and traits. Proc Natl Acad Sci USA. 2009. https://doi.org/10.1073/pnas.0903103106.

3. Khurana E, et al. Role of non-coding sequence variants in cancer. Nat Rev Genet. 2016. https://doi.org/10.1038/ nrg.2015.17.

4. Dunham I, et al. An integrated encyclopedia of DNA elements in the human genome. Nature. 2012. https://doi. org/10.1038/nature11247.

5. Bernstein BE, et al. The NIH roadmap epigenomics mapping consortium. Nat Biotechnol. 2010. https://doi. org/10.1038/nbt1010-1045.

6. Backenroth D, et al. FUN-LDA: a latent Dirichlet allocation model for predicting tissue-specific functional effects of noncoding variation: methods and applications. Am J Hum Genet. 2018. https://doi.org/10.1016/j. ajhg.2018.03.026.

7. Lu Q, et al. Integrative tissue-specific functional annotations in the human genome provide novel insights on many complex traits and improve signal prioritization in genome wide association studies. PLoS Genet. 2016. https://doi.org/10.1371/journal.pgen.1005947.

8. Ionita-Laza I, et al. A spectral approach integrating functional genomic annotations for coding and noncoding variants. Nat Genet. 2016;1:1. https://doi.org/10.1038/ng.3477.

9. Lee $D$, et al. A method to predict the impact of regulatory variants from DNA sequence. Nat Genet. 2015;1:1. https://doi.org/10.1038/ng.3331.

10. Kircher $\mathrm{M}$, et al. A general framework for estimating the relative pathogenicity of human genetic variants. Nat Genet. 2014. https://doi.org/10.1038/ng.2892.

11. Quang D, et al. DANN: a deep learning approach for annotating the pathogenicity of genetic variants. Bioinformatics. 2015. https://doi.org/10.1093/bioinformatics/btu703.

12. Zhou J, Troyanskaya OG. Predicting effects of noncoding variants with deep learning-based sequence model. Nat Methods. 2015. https://doi.org/10.1038/nmeth.3547.

13. Liu Q, et al. Interactive and incremental learning via a mixture of supervised and unsupervised learning strategies. Proc Joint Conf Inf Sci. 2000;1:555-8.

14. Zhu X. Semi-supervised learning literature survey. Technical report 1530, Computer Sciences, University of Wisconsin-Madison 2005

15. Joachims T. Transductive inference for text classification using support vector machines. In: Proceedings of the 20th international conference on machine learning; 2000. p. 200-9.

16. Shental N, et al. Computing Gaussian mixture models with EM using equivalence constraints. Adv Neural Inf Process Syst. 2004;16:465-72.

17. Lee D-H. Pseudo-label: the simple and efficient semi-supervised learning method for deep neural networks. In: ICML 2013 workshop: challenges in representation learning; 2013.

18. Iscen A, et al. Label propagation for deep semi-supervised learning. In: Proceedings of the IEEE computer society conference on computer vision and pattern recognition; 2019. https://doi.org/10.1109/CVPR.2019.00521.

19. Li Z, et al. Naive semi-supervised deep learning using pseudo-label. In: Peer-to-peer networking and applications; 2019. https://doi.org/10.1007/s12083-018-0702-9

20. He Z, et al. A semi-supervised approach for predicting cell-type specific functional consequences of non-coding variation using MPRAs. Nat Commun. 2018. https://doi.org/10.1038/s41467-018-07349-w. 
21. Boyle AP, et al. High-resolution mapping and characterization of open chromatin across the genome. Cell. 2008. https://doi.org/10.1016/j.cell.2007.12.014.

22. Srivastava N, et al. Dropout: a simple way to prevent neural networks from overfitting. J Mach Learn Res. 2014;15:1929-58.

23. Glorot X, et al. Deep sparse rectifier neural networks. J Mach Learn Res. 2011;15:315-23.

24. Ioffe $S$, Christian S. Batch normalization: accelerating deep network training by reducing internal covariate shift. In: 32nd international conference on machine learning, ICML 2015; 2015. p. 448-56.

25. Bottou L. Large-scale machine learning with stochastic gradient descent. In: Proceedings of COMPSTAT 2010 - 19th international conference on computational statistics; 2010. https://doi.org/10.1007/978-3-7908-2604-3-16.

\section{Publisher's Note}

Springer Nature remains neutral with regard to jurisdictional claims in published maps and institutional affiliations.

- fast, convenient online submission

- thorough peer review by experienced researchers in your field

- rapid publication on acceptance

- support for research data, including large and complex data types

- gold Open Access which fosters wider collaboration and increased citations

- maximum visibility for your research: over 100M website views per year

At BMC, research is always in progress.

Learn more biomedcentral.com/submissions 